surgery or radiotherapy were of no benefit. Seizures were refractory to AEDs, only 11 of 164 patients achieving a 1 year remission. The control of tumor-related epilepsy poses a special problem, requiring more specific AEDs. (See Progress in Pediatric Neurology II, PNB Publ, 1994;pp344-345).

Progressive myoclonus in a 22-month-old boy with a deep cerebellar ganglioglioma is reported from the University of Rochester, NY (Mink JW et al. Neurology 2003;61:829-831). The authors theorize that myoclonus resulted from abnormal paroxysmal output from cerebellar nuclei, and was not a case of "cerebellar epilepsy."

The impact of epilepsy and AED treatment on cognitive functioning was studied in 156 patients with low-grade gliomas. (Klein M et al. Ann Neurol 2003;54:514-520). Of $86 \%$ with epilepsy, $50 \%$ of those on AEDs were seizure-free. Compared to healthy controls, glioma patients had significant reductions in psychomotor function, attention, memory, and quality of life. Cognitive dysfunction was attributed to adverse effects of AEDs, whereas decline in quality of life was ascribed to incomplete seizure control.

\title{
CHRONIC EPILEPSY AND COGNITION
}

Cognition, change of memory and nonmemory functions, in 147 surgically and 102 medically treated patients with temporal lobe epilepsy was evaluated in a longitudinal study at the University of Bonn, Germany, and the University of Sheffield, UK. Patients were tested at baseline (T1) and after 2 to 10 years (T3). Surgical patients were also retested 1 year postoperatively (T2). Higher baseline test performance was predictive of better long-term performance and better cognitive outcome. Complete seizure control was obtained in $63 \%$ of surgical and $12 \%$ of medically treated patients. Significant decline in memory (with little change in nonmemory) function had occurred at T3 follow-up in 50\% of medical and $60 \%$ of surgical patients. More limited surgical resections were associated with better cognitive outcome and less risk of cognitive decline. Seizure-free surgical patients showed recovery of nonmemory at T2 $(\mathrm{p}<0.001)$, and of memory functions at T3 $(p=0.03)$. Psychosocial outcome was better when seizures were controlled. (Helmstaedter C, Kurthen M, Lux S, Reuber M, Elger CE. Chronic epilepsy and cognition: a longitudinal study in temporal lobe epilepsy. Ann Neurol October 2003;54:425-432). (Respond: Dr Helmstaedter, University of Bonn, Department of Epileptology, Sigmund Freud Strasse 25, 53105 Bonn, Germany).

COMMENT. Both surgically and medically teated patients with temporal lobe epilepsy (TLE) are at risk of cognitive decline. The degree of cognitive decline is inversely related to seizure control. Surgically treated compared to medically treated patients have better seizure control. When unsuccessful, however, surgery may accelerate the cognitive decline seen with failed medical treatment, especially with left sided temporal lobe resections. When seizures are controlled, either medically or surgically, memory decline is halted or even reversed. Nonmemory functions, related to brain regions distant from the focus, recover more quickly than memory deficits directly associated with the focus. Early and complete control of seizures is necessary to prevent cognitive impairment and adverse effects on quality of life and learning. Memory outcome and that of school or work performance are interrelated, emphasizing the importance of early seizure control. (See Editorial. Duncan JS, Thompson PJ. Ann Neurol 2003;54:421-422). 
The cognitive and behavioral effects of chronic epilepsy have been emphasized in several publications by Deonna T (cited in Deonna T. Childhood epilepsy: secondary prevention is crucial. Dev Med Child Neurol Aug 2003;45 (suppl 95):38-41). Some focal epilepsies or purely EEG paroxysmal discharges without clinical seizures can have a chronic adverse effect on cognition and behavior. Treatment should sometimes be directed not only to the control of clinical seizures but also the prevention of cognitive impairments related to focal epileptiform discharges, as for example, epilepsy with so-called benign rolandic spikes. The risks of cognitive decline related to chronic AED treatment are increasingly apparent, especially with temporal lobe epilepsies, emphasizing the importance of early referral of selected patients to our surgical colleagues.

\section{PROGNOSTIC VALUE OF EEG IN FOCAL SEIZURE CONTROL}

Patients aged 2 to 85 (mean 32) years whose focal seizures were uncontrolled (U) (more than 2 seizures per month) and a group with controlled seizures (C) (fewer than 2 seizures per year), 150 in each group, were randomly selected from files at the University of Illinois Medical Center, Chicago, and EEGs in the two groups (804 in U group and 674 in $\mathrm{C}$ group) were examined for their predictive value of seizure control. Patients with generalized epilepsy and benign epilepsies of childhood were excluded. Younger patients (5-18 yrs) were generally better controlled than those 21-48 years of age, and seizure intractability became more prevalent in the 20 to 40 year age group. Focal spikes and focal slow waves were more common in $\mathrm{U}$ patients than $\mathrm{C}$ patients. Many spikes or slow waves occurred in EEGs of $\mathrm{U}$ patients and no or rare spikes or slow waves in C patients' records. Predictors of $\mathrm{C}$ focal seizures were no or rare spikes, especially on the first or second EEG. $\mathrm{U}$ patients were correctly identified by many spikes (at least one/10 sec) at any location or frontal spikes in $84 \%$ cases ( $61 \%$ with 1 st or 2 nd EEG), but $29 \%\left(21 \%\right.$ in $1^{\text {st }}$ or $\left.2^{\text {nd }} E E G s\right)$ were incorrectly identified as $U$ and seizures were controlled. The EEG is a useful predictor of prognosis of the uncontrolled focal seizure patient, and should be a guide to early more effective therapy. (Hughes JR, Fino JJ. Focal seizures and EEG: prognostic considerations. Clin Electroencephalogr October 2003;34:174-181). (Reprints: John R Hughes MD, PhD, University of Illinois Medical Center M/C 796, $912 \mathrm{~S}$ Wood St, Chicago, IL 60612).

COMMENT. Based on frequent spike occurrence in first or second EEG recordings in patients with focal seizures, a positive prediction of uncontrolled seizures and poor prognosis is likely in $61 \%$ but a false positive may occur in $21 \%$. In any EEG recording, the true positives are $84 \%$ and false positives $29 \%$. Seizure intractability is especially frequent in the 20 to 40 year age group. Patients with rare spikes have less frequent less severe focal seizures and a lower incidence of secondarily generalized tonicclonic seizures (Rosati A et al. Neurology 2003;60:1290-1295; cited by Hughes). 